\title{
The Seminal Plasma of the Boar is Rich in Cytokines, with Significant Individual and Intra-Ejaculate Variation
}

Isabel Barranco, Marie Rubér, Cristina Perez-Patino, Mohammad Atikuzzaman, Emilio A.

Martinez, Jordi Roca and Heriberto Rodriguez-Martinez

\section{Linköping University Post Print}

\section{Tweet}

N.B.: When citing this work, cite the original article.

Original Publication:

Isabel Barranco, Marie Rubér, Cristina Perez-Patino, Mohammad Atikuzzaman, Emilio A. Martinez, Jordi Roca and Heriberto Rodriguez-Martinez, The Seminal Plasma of the Boar is Rich in Cytokines, with Significant Individual and Intra-Ejaculate Variation, 2015, American Journal of Reproductive Immunology, (74), 6, 523-532.

http://dx.doi.org/10.1111/aji.12432

Copyright: Wiley: 12 months

http://eu.wiley.com/WileyCDA/

Postprint available at: Linköping University Electronic Press

http://urn.kb.se/resolve?urn=urn:nbn:se:liu:diva-124497 


\section{The seminal plasma of the boar is rich in cytokines, with significant individual and intra-ejaculate variation}

Isabel Barranco', Marie Rubér², Cristina Perez-Patiño', Mohammad Atikuzzaman², Emilio A. Martinez ${ }^{1}$, Jordi Roca ${ }^{1}$, Heriberto Rodriguez-Martinez ${ }^{2}$

${ }^{1}$ Dpt. Medicine \& Animal Surgery, University of Murcia, Murcia, Spain.

${ }^{2}$ Dpt. Clinical \& Experimental Medicine, University of Linköping, Linköping, Sweden

\section{Correspondence:}

Jordi Roca, Department of Medicine and Animal Surgery, Faculty of Veterinary Science, University of Murcia, E-30100 Murcia, Spain. E-mail: roca@um.es. Tel.: +34 868884735.

Running head: Cytokines in boar seminal plasma 


\section{Abstract}

Problem: The boar, as human, sequentially ejaculates sperm-rich and sperm-poor fractions. Seminal plasma (SP) spermadhesins (PSP-I/PSP-II) induce a primary endometrial inflammatory response in female sows, similar to that elicited by semen deposition in other species, including human. However, the SP is also known to mitigate such response, making it transient to allow for embryo entry to a cleansed endometrium. Although cytokineinvolvement has been claimed, the exploration of cytokines in different SP-fractions is scarce. This study determines Th1, Th2, Th17 and Th3 cytokine profiles in specific ejaculate SP-fractions from boars of proven fertility.

Methods: SP-samples from the sperm rich fraction (SRF) and the sperm-poor post-SRF fractions (post-SRF) of manually-collected ejaculates from eight boars (four ejaculates per boar) were analyzed by commercial multiplex bead assay kits (Milliplex MAP, Millipore USA) for interferon- $\gamma$, interferon gamma-induced protein 10, macrophage-derived chemokine, growth-regulated oncogene, granulocyte-macrophage colony-stimulating factor, monocyte chemoattractant protein-1, interleukins (IL)-6, IL-8, IL-10, IL-15, IL-17 and transforming growth factor (TGF)- $\beta 1-\beta 3$.

Results: Cytokine concentrations differed between the ejaculate fractions among boars, being highest in the post-SRF.

Conclusions: Boar SP is rich in Th1, Th2, Th17 and Th3 cytokines, with lowest concentrations in the sperm peak-containing fraction, indicating its main immune influence might reside in the larger, protein-rich sperm-poor post-SRF.

\section{Keywords:}

Ejaculate fractions, immunomodulatory molecules, seminal plasma peptides, pig. 


\section{Introduction}

Seminal plasma (SP), the heterogenous composite fluid where spermatozoa are suspended during ejaculation, is mainly composed by secretions of the male accessory sexual glands (in pigs; seminal vesicles, prostate and bulbo-urethral/urethral glands), but also contains caudae epididymides intraluminal fluid that accompanies the emitted spermatozoa. ${ }^{1}$ In pigs, the large $(150-250 \mathrm{~mL})$ ejaculate is expelled in three time-sequenced fractions, which differ in sperm numbers and SP quantity and composition. The first fraction is the so-called watery presperm fraction that precedes sperm emission (urethral/bulbourethral secretion). The following fraction is conspicuous, called sperm-rich fraction (SRF) because it contains most (above $80 \%$ ) of spermatozoa suspended in cauda epididymal fluid and prostate secretion, with a minor portion of seminal vesicle secretion. Following this dense, $30-50 \mathrm{~mL}$ large fraction, the rest of the non-coagulating ejaculate is called post-SRF, containing much fewer spermatozoa in a larger (often 120-200 mL) amount of SP mainly derived from the seminal vesicles, although secretion from the prostate gland is rather constant during the entire ejaculate and thus also present in this fraction. ${ }^{2}$ A coagulating end-secretion, with typical tapioca-like gel balls closes the ejaculation cycle, these secretions being formed in the bulbourethral glands and aimed at coagulating the protein-rich last part of the ejaculate to plug the cervical canal. ${ }^{1}$ Semen collection in pigs is routinely done using manual pressure on the glans penis (the so-called gloved-hand method) and allows for separate collection of the various fractions during ejaculation. As such, the SRF is most often retrieved for preparation of artificial insemination (AI) semen doses and for other sperm processing technologies such as freezing and sex-sorting. ${ }^{3,4}$ The SP affects sperm function, demonstrated both in vivo and 
in vitro studies with a focus on SP-proteins ${ }^{5,6,7}$. For instance, spermadhesins such as AWN1 bind to the sperm plasmalemma and follow them to the oocyte, influencing the process of sperm capacitation and sperm-oocyte binding. ${ }^{8}$ The SP is also able to mitigate the initial inflammatory response elicited by semen deposition, restricting this to a cleansing transient state preparing the endometrium for the descending early embryos. ${ }^{9}$ Even the SP of specific fractions or portions within the SRF (the so-called sperm-peak fraction) seem to conspicuously affect the functionality and survival of ejaculated, processed (chilled or frozen-thawed spermatozoa), thus indicating the sequential ejaculation process has physiological implications, at least in vivo. ${ }^{10}$ In commercial enterprises of boar semen production for AI, boar ejaculates are now being collected using semi-automatic collecting devices which collect the entire ejaculate in one single flask, thus obtaining a nonphysiological mixture of SP components. The impact of this practice is yet unknown.

The pig SP is particularly rich in proteins $(30-40 \mathrm{~g} / \mathrm{L}$, most of this contents present in the post-SRF ${ }^{11,12}$ ) but also in peptides, ions, energy substrates, buffer substances, amino acids, lipids and hormones. ${ }^{5,13}$ These SP-proteins participate in key events related to fertilization, from participation in the sperm transport through the female genitalia, the establishment of the oviductal sperm reservoir, sperm capacitation and gamete interaction. ${ }^{14}$ Moreover, SPproteins and peptides act as signals for the female immune system, modulating the maternal tolerance towards embryo and placental development, thus conditioning embryo development in eutherian mammals, including human..$^{5}$ Among the latter are cytokines, secreted by activated immune cells and male/female genital epithelia, including that of male sexual accessory glands or of the vagina, cervix and uterus. ${ }^{15,16}$ Cytokine levels in SP have, 
moreover, associated with poor sperm quality or functionality, and to sub-fertility in men with or without pathological processes in their genital tract. ${ }^{17-19}$

In the case of the boar, a species whose fractionated ejaculate sequence mimics that of human, the proteome of the ejaculate is rather well known. ${ }^{5}$ Yet, the peptidome is less screened. 5,6 For instance, to the best of our knowledge, only few cytokines have been identified in boar SP, namely the transforming growth factor- $\beta$ (TGF- $\beta$ ) 1 and 2 , interferon- $\gamma$ (IFN- $\gamma$ ) and interleukins (IL)-6 and IL-10..$^{20,21}$ with clear quantitative variation between ejaculate fractions. In comparison, many other cytokines, e.g IL-6, IL-8, IL-10, IL-11, IL-23 and IFN$\gamma$, have been found in human ejaculates, some of them displaying either positive or negative

correlations with sperm quality and fertility. ${ }^{22,23}$ Whether the boar SP possess a similarly full battery of cytokines remains to be explored.

The purpose of the present study was, therefore, to identify and quantify a representative battery of measurable cytokines in the most conspicuous fractions of the boar SP, covering Th1, Th2, Th17 and Th3 -related cytokine responses.

\section{Material and methods}

\section{Animals and ejaculates}

All procedures involving animals were performed according to international guidelines and were approved by the Bioethics Committee of Murcia University (research code: 639/2012). Eight healthy, fertility-proven boars of different breeds or crossbreds were used as semen providers. Boars were housed in individual pens in an AI center (AIM Iberica, Calasparra, Murcia, Spain), environmentally controlled $\left(15-25^{\circ} \mathrm{C}\right)$ with windows exposed to natural 
daylight and supplementary light for a total of $16 \mathrm{~h}$ of light per day. Boars were provided with ad libitum access to water and were fed commercial feedstuff according to the nutritional requirements for adult boars subjected to regular ejaculate collection. ${ }^{24}$ Thirty-two ejaculates (four per boar) were manually collected fraction-wise, namely separating the SRF and the following post-SRF, using the gloved-hand method. All collected ejaculates fulfilled the standards of quantity and sperm quality thresholds for the preparation of AI-semen doses (more than $200 \times 10^{6}$ spermatozoa/mL, $70 \%$ of them motile and $75 \%$ depicting with normal morphology).

\section{Seminal plasma processing and storage}

Aliquots of each ejaculated SRF and post-SRF were immediately transferred to $15 \mathrm{~mL}$ tubes and centrifuged twice (Rotofix 32A; Hettich Zentrifugen, Tuttlingen, Germany) at 1,500g for $10 \mathrm{~min}$ at room temperature (RT). The second supernatant was, after being examined by light microscopy to ensure it was sperm-free, transferred to $3 \mathrm{~mL}$-cryotubes. The SP-samples were transported within $2 \mathrm{~h}$ of collection in insulated containers $\left(15-17{ }^{\circ} \mathrm{C}\right)$ to the Andrology

Laboratory at the Veterinary Teaching Hospital of the University of Murcia (Spain) and frozen $\left(-80{ }^{\circ} \mathrm{C}\right)$ until isothermically-shipped for cytokine determination at the Department of Clinical \& Experimental Medicine, Linköping University, Linköping (Sweden).

\section{Measurement of cytokines in boar seminal plasma}

The Luminex's xMAP® technology, a multiplexed microsphere-based flow cytometric assay, was used to examine the presence and relative concentration of a battery of cytokines and chemokines including IL-6, IL-8, the monocyte chemoattractant protein-1 (MCP-1) or the growth factor granulocyte macrophage colony-stimulating factor (GM-CSF) (associated 
with a pro-inflammatory immune response); the anti-inflammatory/immune deviating, Th3associated IL-10 and TGF- $\beta 1$, TGF- $\beta 2$ and TGF- $\beta 3$; the Th1-associated IFN- $\gamma$ and interferon gamma-induced protein (IP-10/CXCL10); the Th2-associated macrophage-derived chemokine (MDC); the Th17-associated IL-17 and growth-regulated oncogene (GRO/CXCL1); as well as the inducer of NK cell proliferation and IL-15.

Pre-coated magnetic beads (Cat\#HCYTOMAG-60K-11 for human reactivity, Merck Millipore, Billerica, MA, USA) were used for the determination of IL-6, IL-8, MCP-1, IL10, IFN- $\gamma$, IP-10/CXCL10, MDC, IL-17, GRO/CXCL1, GM-CSF, and IL-15; while for quantification of TGF- $\beta 1$, TGF- $\beta 2$ and TGF- $\beta 3$, a 3-plex kit (Cat\#TGFB-64K-03 for pig, human, mouse, rat, non-human primate, canine, feline reactivity, Merck Millipore) were used, following the methods described by the manufacturers in 96-well multiscreen plates. A cytokine standard curve, comprising six standard points, was built for each cytokine with the highest standard point at $10,000 \mathrm{pg} / \mathrm{mL}$ and the lowest standard point at $3.2 \mathrm{pg} / \mathrm{mL}$, while for TGF- $\beta$ the highest point was $10,000 \mathrm{pg} / \mathrm{mL}$ and the lowest $9.8 \mathrm{pg} / \mathrm{mL}$. Serum matrix (SM), provided in the kits, was used to mimic the composition of the seminal environment in the standard, control and blank measurements. Two controls provided in the kits, were added in singlets. Following sonication, bead solution was added to each well for incubation at $4{ }^{\circ} \mathrm{C}$ in the dark, for $18 \mathrm{~h}$. After incubation, the plate was emptied using a multiscreen vacuum manifold (Merck Millipore), washed twice, detection antibody added, incubated at RT in darkness for $60 \mathrm{~min}$, before streptavidin-phycoerythrin addition and further incubated for 30 min. After washing, the plates were run on a Luminex 200 TM (Luminexcorp, Austin, TX, USA) with xPONENT software version 3.1.7 (Luminex corp) for acquisition and Masterplex 2010 version 2.0.0.68 (Mirai Bio Group, San Francisco, CA, USA) for data analysis. The median fluorescent intensity was analysed using a 5-parameter logistic curve-fitting to 
calculate the concentrations of the cytokines in the samples. The samples for TGF- $\beta$ were acidified ( $\mathrm{pH}<3$ ) with $8 \mu \mathrm{L}$ of $\mathrm{HCl}$, and processed as described above for the cytokines and chemokines, except that the samples were diluted 1:30 v/v with $\mathrm{xxx}$ before analyses.

The data of cytokine concentration are shown for each ejaculate fraction (SRF and post-SRF) as means $( \pm$ SEM $)$, median and percentiles.

\section{Statistical analysis}

Statistical analyses were carried out using the SPSS Statistics version 19 (IBM SPSS Statistics, Chicago, IL, USA). Normal distribution of residuals was tested using Kolmogorov-Smirnov test. The variation in concentrations of cytokines among boar and ejaculate fractions was analysed using mixed models of ANOVA, including ejaculate or ejaculate-fraction (four ejaculates per boar) as random effect. A $P$-value $<0.05$ was considered to be statistically significant.

\section{Results}

A total of 14 measurable cytokines were identified in the SP of the two main boar ejaculate fractions. With the exception of TGF- $\beta 3$, only measurable in the SP of 4 boars, all other cytokines explored were quantifiable in the SP of all boars (Figures 1-3). While the cytokines representative of Th1, Th2, Th3 and Th17 responses showed measurable concentrations in the SP from the post-SRF, three of them, GRO/CXCL1, IL-8 and IL-15, did not show measurable concentrations in the SP from SRF (Figures 1 and 2). The measurable concentrations of all of them varied significantly $(P<0.001)$ between boars. For those 
cytokines detectable in either fraction of the ejaculate, their concentrations differed, with the post-SRF invariably showing the highest $(P<0.05)$ concentrations (Figures $1-3)$.

\section{Discussion}

To the best of our knowledge, this is the first study measuring cytokines representative of the Th1, Th2, Th3 and Th17 response groupings in the SP of a major livestock species; the pig, far beyond the five known to date. ${ }^{20,21}$ Such large number of cytokines (e.g. 14) had only been identified so far in human and murine SP, which had been compared as whole ejaculate despite having dissimilar anatomy, physiology, ejaculate composition and type of ejaculate. $^{25,26}$ In human, cytokine type and concentrations have been related to the normality or (in) fertility of the males examined. For instance, high concentrations of IFN- $\gamma$, IL-17, MCP-1, MDC, IL-6 and IL-8 had been measured in SP-samples from infertile men or having pathological processes in their genital tract. ${ }^{17-19,27,28}$ Some other SP-cytokines, such as IL-10, had shown contradictory results among studies. Huleihel et al. (1999) reported lower concentrations of IL-10 in infertile than fertile men, whereas Miller et al. (2002) reported opposite results. ${ }^{29,30}$ These conflicting results, together with the little current knowledge of the role played by SP-cytokines on male reproductive performance, calls for further studies. Comparatively, the concentrations of IP-10/CXCL10, GRO/CXCL1, MCP-1 and TGF- $\beta 2$ seemed higher in boar SP than in human SP. ${ }^{16,31,32}$ In contrast, the concentration of INF- $\gamma$, MDC, IL-8 and TGF- $\beta 3$ are apparently lower in boar SP than in human SP. ${ }^{16,19,23}$ Whether these differences simply represent species differences or whether they have a relationship to physiological events in the male or the female, remains to be determined. 
A main purpose of our study was to detect and measure the cytokine IL-15 in SP. This cytokine is a member of the four- $\alpha$ helix bundle cytokine family, which acts as a T-cell stimulant and plays a pivotal role in cell-mediated immunity by activating T-cell proliferation and B-cell antibody production, and by promoting natural killer cell cytotoxicity. ${ }^{33,34}$ Moreover, IL-15 has been demonstrated to play a relevant role in uterine function, acting through IL-15 endometrium receptors during implantation and early placental development. ${ }^{34}$ In addition, it has been suggested that IL-15 is involved in the regulation of metrial gland function during mouse pregnancy. ${ }^{35}$

The origin and particularly the role played by the above measured SP-cytokines on the male or female reproductive tract are far from clear. Regarding their origin, previous studies suggested that cytokines as GRO/CXCL1 and IP-10/CXCL10 can be synthesized by somatic cells in the seminiferous tubules (Sertoli cells and myoid cells). ${ }^{36}$ However, the bulk of SPcytokines is mainly synthetized by the accessory sexual glands. ${ }^{37-39}$ The prostate in humans and the seminal vesicles in mice are the main source of the latent isoforms of TGF- $\beta$, while a comparative origin in the pig can only be indirectly related to the origin of the fluids in every fraction. ${ }^{37,38}$ In that respect, both prostate and seminal vesicle secretions are primary contributors. Since the SP of SRF comes mainly from the prostate and epididymides and less from seminal vesicles while the SP of the post-SRF comes mainly from seminal vesicles (with a testimonial contribution of bulbourethral glands), it may be speculated that the seminal vesicles could be the main origin for many cytokines. ${ }^{2}$ However, it is also reasonable to hypothesize that the three isoforms of TGF- $\beta$, present in both ejaculate fractions, could be synthetized by both prostate and seminal vesicles, but that the acidic SRF fraction (owing to the acidic cauda epididymis fluid ) might be most relevant for the activation of the cytokine in the boar. ${ }^{40}$ It is less likely that extravasation from blood to the SP is as alternative major 
mechanism, particularly considering the boar has a membranous type of penis where blood flow (dramatically increased in humans for instance) is not noticeably increased during ejaculation $^{41}$

Moreover, cytokines secreted by epididymal and accessory sexual glands can be released to the SP within or in relation to exosomes which, either of epididymal (epididymosomes) or prostatic (prostasomes) origin, depict immunomodulatory properties. ${ }^{42,43}$ The SP-cytokines, independently of their origin, interact with the epithelia of the female genitalia which they confront during semen deposition. Such interaction can occur via exposure of SP directly to the epithelium, or through their binding to the sperm surface (as in the case of TGF- $\beta$, which binds to the post-acrosome domain in human). ${ }^{44}$ Several SP-components, apart from bacterial lipopolysaccharides (LPS), TGF- $\beta$, IL-8 etc., are able to induce epithelial production of cytokines. For instance TGF- $\beta$ induces epithelial production of GM-CSF in mice and human. ${ }^{37,39}$ IFN- $\gamma$ is, on the contrary, capable of inhibiting the TGF- $\beta$ signaling of epithelial cells. ${ }^{45}$ Considering all above information, the transient inflammatory response of the female genitalia caused by semen deposition that occurs in all mammals studied so far after seems to be dual, elicited by spermatozoa and SP proteins. ${ }^{5}$ Moreover, SP-cytokines and those produced by the responding female genital epithelium seem also to participate. However, this inflammation resumes, often within hours, and information is available indicating the presence of TGF- $\beta$ elicits -via epithelial production of GM-CSF- a monocytic transformation into macrophages within the endometrial epithelium and its subyacent lamina propria. ${ }^{9}$ These macrophages would further migrate via the lymphatic system to other areas, modify the immune response, terminating the inflammatory primary response and initiate a state of immunological tolerance towards implantation and further embryo development. ${ }^{39,46,47}$ Initially, it was believed that the porcine SP had anti-inflammatory properties, decreasing the 
immune response in the uterus, thereby facilitating the sperm transport in the female genital tract and the colonization of the utero-tubal junction. ${ }^{48}$ However, the current results show that the boar SP contains cytokines with pro- and anti-inflammatory properties, which confirms the dual SP-effect above described for in human and mice, would also appear in sows. Such effect was partially demonstrated by Jalali et al.,(2014) who asserted that the SP produces an increase in Treg-immune cells and a decrease in expression of inflammatory cytokines in the sow endometrium. ${ }^{49}$

The current study also provides clear evidence of differences among boars in both presence and concentration of some cytokines. Differences in concentration were expected since it is well known that SP-composition differs quantitatively and qualitatively among boars, probably genomically-driven. ${ }^{50,51}$ It is particularly interesting that the cytokine TGF- $\beta 3$ was not measurable in SP-samples of four of the eight boars analyzed and that the concentrations clearly differed between porcine and human. ${ }^{39}$ Noteworthy, these four boars also showed low concentrations of TGF- $\beta 1$. Considering the three isoforms of TGF- $\beta$ have impact on fertility through mediation of maternal immune tolerance after implantation, this finding initiates the question: are these levels related to male (or female) fertility after insemination? ${ }^{20,52}$ It could, for instance, be speculated that boars with absence of TGF- $\beta 3$ together with low concentrations of TGF- $\beta 1$ in their SP may have low reproductive performance. This would be challenged in further studies, pertaining novel preliminary findings (Barranco et al., unpublished data) that the SP of high-fertility boars showed consistent amounts of TGF- $\beta$ over successive ejaculates, compared to highly variable inter-ejaculate concentrations in boars with significantly lower fertility. Further studies of a larger number of males with clear differences in fertility after AI are obviously needed. 
An peculiarity of the ejaculate of boar (and of man) is that it provides two fractions with significant differences in sperm number and proportion of SP that are objectively distinguishable during the traditional manual collection of ejaculates. With the exception of the three isoforms of TGF- $\beta$, the concentrations of the other measurable cytokines differed between fractions. The post-SRF, which contains fewer spermatozoa bathing in larger volumes of SP, showed the highest cytokine concentrations in all boars examined. Moreover, some cytokines, specifically GRO/CXCL1, IL-8 and IL-15 could only be detected in the post-SRF fraction. For semen handling purposes (AI, semen freezing etc) manual collection of the SRF, containing most spermatozoa in a relatively low quantity of SP, has been customary. However, there is an increasing use of semi-automatic devices for entire-ejaculate collection, a practice that lower labor costs for the AI-enterprises. ${ }^{53}$ This change not only implies the sperm suspension is greatly extended into a non-physiologically mixed-SP, deviating from the SP-composition encountered in vivo or when manual fractionated semen collection is done. Whether the different distribution and amount of cytokines influences the reproductive performance of boar ejaculates after AI should be explored. In addition, considering that the specific types and amounts of SP cytokines seem to contribute to the endometrial preparation for embryo implantation, we could hypothesize that avoiding the use of specific fractions may have an implication on resulting fertility rates. ${ }^{16}$

In conclusion, boar seminal plasma is rich in cytokines, whose concentrations differ among boars and also between the two main ejaculate fractions, showing the post-sperm rich fraction (post-SRF) had the highest concentrations. Further studies to elucidate the role of these cytokines on the reproductive performance of boar ejaculates are needed.

\section{Acknowledgements}


This experimental study was supported by MINECO (AGL2012-39903) Madrid (Spain), FEDER funds (EU) and Formas (Stockholm, Sweden). I. Barranco and C. Perez-Patiño were financially supported by MECD (Madrid, Spain) and Seneca Foundation (Murcia, Spain), respectively. The authors are grateful to AIM Iberica for supplying the boar ejaculates.

\section{References}

1. Mann T, Lutwak-Mann C: Male Reproductive Function and Semen. First edition. New York, Springer-Verlag, Berlin Heidelberg, 1981.

2. Einarsson S: Studies on the composition of epididymal content and semen in the boar. Acta Vet Scand Suppl 1971; 36:1-80.

3. Juarez JD, Parrilla I, Vazquez JM, Martinez EA, Roca J: Boar semen can tolerate rapid cooling rates prior to freezing. Reprod Fertil Dev 2011; 23:681-690.

4. Alkmin DV, Parrilla I, Tarantini T, Parlapan L, Del Olmo D, Vazquez JM, Martinez EA, Roca J: Intra- and interboar variability in flow cytometric sperm sex sorting. Theriogenology 2014; 82:501-508.

5. Rodriguez-Martinez H, Kvist U, Ernerudh J, Sanz L and Calvete JJ: Seminal plasma proteins: what role do they play? Am J Reprod Immunol 2011; 66:11-22.

6. Caballero I, Vazquez JM, García EM, Parrilla I, Roca J, Calvete JJ, Sanz L, Martínez EA: Major proteins of boar seminal plasma as a tool for biotechnological preservation of spermatozoa. Theriogenology 2008; 70:1352-1355.

7. Okazaki T, Akiyoshi T, Kan M, Mori M, Teshima H, Shimada M: Artificial insemination with seminal plasma improves the reproductive performance of frozen-thawed boar epididymal spermatozoa. J Androl 2012; 33:990-998. 
8. Calvete JJ, Ensslin M, Mburu J, Iborra A, Martínez P, Adermann K, Waberski D, Sanz L, Töpfer-Petersen E, Weitze KF, Einarsson S, Rodríguez-Martínez H. Monoclonal antibodies against boar sperm zona pellucida-binding protein AWN-1. Characterization of a continuous antigenic determinant and immunolocalization of AWN epitopes in inseminated sows. Biol Reprod 1997; 57:735-742

9. Rozeboom KJ, Troedsson MH, Hodson HH, Shurson GC, Crabo BG: The importance of seminal plasma on the fertility of subsequent artificial inseminations in swine. J Anim Sci $2000 ; 78: 443-448$.

10. Saravia F, Wallgren M, Johannisson A, Calvete JJ, Sanz L, Peña FJ, Roca J, RodríguezMartínez H: Exposure to the seminal plasma of different portions of the boar ejaculate modulates the survival of spermatozoa cryopreserved in MiniFlatPacks. Theriogenology 2009; 71:662-675.

11. Rodriguez-Martinez H, Kvist U, Saravia F, Wallgren M, Johannisson A, Sanz L, Peña FJ, Martinez EA, Roca J, Vazquez JM, Calvete JJ: The physiological roles of the boar ejaculate. In Control of Pig Reproduction VIII, H Rodriguez-Martinez, JL Vallet, AJ Ziecik (eds). UK, Nottingham University Press, 2009, pp 1-21.

12. Novak S, Ruiz-Sanchez A, Dixon WT, Foxcroft GR, Dyck MK: Seminal plasma proteins as potential markers of relative fertility in boars. J Androl 2010; 31:188-200.

13. Juyena NS and Stelletta C: Seminal plasma: an essential attribute to spermatozoa. $J$ Androl 2012; 33:536-551.

14. Töpfer-Petersen E, Ekhlasi-Hundrieser M, Kirchhoff C, Leeb T, Sieme H: The role of stallion seminal proteins in fertilisation. Anim Reprod Sci 2005; 89:159-70. 
15. Qian L, Sun G, Zhou B, Wang G, Song J, He H: Study on the relationship between different cytokines in the semen of infertility patients. Am J Reprod Immunol 2011; 66:157-161.

16. Sharkey DJ, Macpherson AM, Tremellen KP, Mottershead DG, Gilchrist RB, Robertson SA: TGF- $\beta$ mediates proinflammatory seminal fluid signaling in human cervical epithelial cells. J Immunol 2012; 189:1024-1035.

17. Naz RK, Kaplan P: Increased levels of interleukin-6 in seminal plasma of infertile men. J Androl 1994; 15:220-227.

18. Eggert-Kruse W, Boit R, Rohr G, Aufenanger J, Hund M, Strowitzki T: Relationship of seminal plasma interleukin (IL) -8 and IL-6 with semen quality. Hum Reprod 2001; $16: 517-28$.

19. Penna G, Mondaini N, Amuchastegui S, Degli Innocenti S, Carini M, Giubilei G, Fibbi B, Colli E, Maggi M, Adorini L: Seminal plasma cytokines and chemokines in prostate inflammation: interleukin 8 as a predictive biomarker in chronic prostatitis/chronic pelvic pain syndrome and benign prostatic hyperplasia. Eur Urol 2007; 51:524-533.

20. O'Leary S, Armstrong DT, Robertson SA: Transforming growth factor- $\beta$ (TGF $\beta$ ) in porcine seminal plasma. Reprod Fertil Dev 2011; 23:748-758.

21. Jiwakanon J, Dalin AM: Short communication: Concentration of TGF- $\beta 1$, IL-10 and IL6 in boar seminal plasma and TGF- $\beta 1$ level in different fractions of ejaculates. Anim Reprod Sci 2012; 131:194-198.

22. Seshadri S, Bates M, Vince G, Jones DI: The role of cytokine expression in different subgroups of subfertile men. Am J Reprod Immunol 2009; 62:275-282.

23. Seshadri S, Bates M, Vince G, Jones DI: Cytokine expression in the seminal plasma and its effects on fertilisation rates in an IVF cycle. Andrologia 2011; 43:378-386. 
24. Chiba LI: Pig nutrition and feeding. In Animal Nutrition Handboock, Chiba LI (eds). Auburn, Auburn University, 2009, pp 285-315.

25. Maegawa M, Kamada M, Irahara M, Yamamoto S, Yoshikawa S, Kasai Y, Ohmoto Y, Gima H, Thaler CJ, Aono T: A repertoire of cytokines in human seminal plasma. $J$ Reprod Immunol 2002; 54:33-42.

26. Gopichandran N, Ekbote UV, Walker JJ, Brooke D, Orsi NM: Multiplex determination of murine seminal fluid cytokine profiles. Reproduction 2006; 131:613-621.

27. Paradisi R, Capelli M, Mandini M, Bellavia E, Flamigni C: Increased levels of interferongamma in seminal plasma of infertile men. Andrologia 1996; 28:157-161.

28. Sabbaghi M, Aram R, Roustaei H, Fadavi Islam M, Daneshvar M, Castaño AR, Haghparast A: IL-17A concentration of seminal plasma and follicular fluid in infertile men and women with various clinical diagnoses. Immunol Invest 2014; 43:617-626.

29. Huleihel M, Lunenfeld E, Horowitz S, Levy A, Potashnik G, Mazor M, Glezerman M: Expression of IL-12, IL-10, PGE2, sIL-2R and sIL-6R in seminal plasma of fertile and infertile men. Andrologia 1999; 31:283-288.

30. Miller LJ, Fischer KA, Goralnick SJ, Litt M, Burleson JA, Albertsen P, Kreutzer DL: Interleukin-10 levels in seminal plasma: implications for chronic prostatitis-chronic pelvic pain syndrome. J Urol 2002; 167:753-756.

31. Hakimi H, Zainodini N, Khorramdelazad H, Kazemi Arababadi M and Hassanshahi G: Seminal Levels of Pro-inflammatory (CXCL1, CXCL9, CXCL10) and Homeostatic (CXCL12) Chemokines in Men With Asymptomatic Chlamydia trachomatis Infection. Jundishapur J Microbiol 2014; 7:e11152. 
32. Politch JA, Tucker L, Bowman FP, Anderson DJ: Concentrations and significance of cytokines and other immunologic factors in semen of healthy fertile men. Hum Reprod $2007 ; 22: 2928-2935$.

33. Carson WE, Giri JG, Lindemann MJ, Linett ML, Ahdieh M, Paxton R, Anderson D, Eisenmann J, Grabstein K, Caligiuri MA: Interleukin (IL) 15 is a novel cytokine that activates human natural killer cells via components of the IL-2 receptor. J Exp Med 1994; 180:1395-1403.

34. Okada S, Okada H, Sanezumi M, Nakajima T, Yasuda K, Kanzaki H: Expression of interleukin-15 in human endometrium and decidua. Mol Hum Reprod 2000; 6:75-80.

35. Allen MP, Nilsen-Hamilton M: Granzymes D, E, F, and G are regulated through pregnancy and by IL-2 and IL-15 in granulated metrial gland cells. J Immunol 1998; $161: 2772-2279$.

36. Aubry F, Habasque C, Satie AP, Jégou B, Samson M: Expression and regulation of the CXC-chemokines, GRO/KC and IP-10/mob-1 in rat seminiferous tubules. Eur Cytokine Netw (2000); 11:690-698.

37. Tremellen KP, Seamark RF, Robertson SA: Seminal transforming growth factor beta 1 stimulates granulocyte-macrophage colony-stimulating factor production and inflammatory cell recruitment in the murine uterus. Biol Reprod 1998; 58:1217-1225.

38. Gann PH, Klein KG, Chatterton RT, Ellman AE, Grayhack JY, Nadler RB, Lee C: Growth factors in expressed prostatic fluid from men with prostate cancer, BPH and clinically normal prostates. Prostate 1999; 40:248-255.

39. Robertson SA: Seminal plasma and male factor signalling in the female reproductive tract. Cell Tissue Res 2005; 322:43-52. 
40. Rodriguez-Martinez H, Ekstedt E, Einarsson S. Acidification of epididymal fluid in the boar. Int J Androl 1990; 13:238-243.

41. Senger PL. Pathways to pregnancy and parturition. Current Conceptions Inc $3^{\text {rd }}$ edition, Pullman WA USA, 2012.

42. Ronquist G: Prostasomes are mediators of intercellular communication: from basic research to clinical implications. J Intern Med 2012; 271:400-413.

43. Piehl LL, Fischman ML, Hellman U, Cisale H, Miranda PV: Boar seminal plasma exosomes: effect on sperm function and protein identification by sequencing. Theriogenology 2013; 79:1071-1082.

44. Chu TM, Nocera MA, Flanders KC, Kawinski E: Localization of seminal plasma transforming growth factor-betal on human spermatozoa: An immunocytochemical study. Fertil Steril 1996; 66:327-330.

45. Glynn DJ, Sharkey DJ, Robertson SA: Interferon-gamma inhibits female reproductive tract responsiveness to seminal plasma. Biol Reprod 2004; 242.

46. Robertson SA, Guerin LR, Moldenhauer LM, Hayball JD: Activating T regulatory cells for tolerance in early pregnancy - the contribution of seminal fluid. $J$ Reprod Immunol 2009; 83:109-16.

47. Bromfield JJ: Seminal fluid and reproduction: much more than previously thought. $J$ Assist Reprod Genet 2014; 31:627-636.

48. Rozeboom KJ, Troedsson MH, Rocha GR, Crabo BG: The chemotactic properties of porcine seminal components toward neutrophils in vitro. J Anim Sci 2001; 79:996-1002.

49. Jalali BM, Kitewska A, Wasielak M, Bodek G, Bogacki M: Effects of seminal plasma and the presence of a conceptus on regulation of lymphocyte-cytokine network in porcine endometrium. Mol Reprod Dev 2014; 81:270-281. 
50. Strzezek J, Wysocki P, Kordan W, Kuklinska M, Mogielnicka M, Soliwoda D, Fraser L: Proteomics of boar seminal plasma - current studies and possibility of their application in biotechnology of animal reproduction. Reprod Biol 2005; 5:279-290.

51. Thurston LM, Siggins K, Mileham AJ, Watson P, Holt WV: Identification of amplified restriction fragment length polymorphism markers linked to genes controlling boar sperm viability following cryopreservation. Biol Reprod 2002; 66: 545-554.

52. Robertson SA, Ingman WV, O'Leary S, Sharkey DJ, Tremellen KP: Transforming growth factor beta a mediator of immune deviation in seminal plasma. J Reprod Immunol 2002; 57:109-28.

53. Barrabes SB, Aneas BG, Gary BP: Bouvier Collectis® automated boar collection technology. Theriogenology 2008; 70:1368-1373.

Figure legends:

Figure 1. Box-whisker plot showing the variation in cytokines and chemokines produced by T helper cells mediating type 1 response (Interferon $\gamma[$ INF- $\gamma]$ and Interferon gamma-induced protein 10 [IP-10/CXCL10]); type 2 response (Macrophage-derived chemokine [MDC]) and type 17 response (Interleukin-17 [IL-17] and Growth-regulated oncogene [GRO/CXCL1]) in the seminal plasma from the sperm-rich fraction (SRF) and the post-sperm fraction, (POSTSRF) of 32 ejaculates from 8 boars ( 4 per boar). The boxes enclose the 25 th and 75 th percentiles; the line is the median; and the whiskers extend to the 5th and 95th percentiles. Data are the means \pm SEM of 4 semen samples per boar. 
Figure 2. Box-whisker plot showing the variation in pro-inflamatory cytokines and chemokines (Interleukin [IL]-6,-8,-15, Granulocyte-macrophage colony-stimulating factor [GM-CSF] and Monocyte chemoattractant protein-1 [MCP-1]) in the seminal plasma from the sperm-rich fraction (SRF) and the post-sperm fraction, (POST-SRF) of 32 ejaculates from 8 boars ( 4 per boar). The boxes enclose the 25 th and 75 th percentiles; the line is the median; and the whiskers extend to the 5th and 95th percentiles. Data are the means \pm SEM of 4 semen samples per boar.

Figure 3. Box-whisker plot showing the variation in cytokines and chemokines produced by T helper cells type 3 response (anti-inflammatory/immunotolerance-related Interleukin [IL]10 and Transforming growth factor $[\mathrm{TGF}]-\beta 1,-\beta 2,-\beta 3)$ in the seminal plasma from the sperm-rich fraction (SRF) and the post-sperm fraction, (POST-SRF) of 32 ejaculates from 8 boars ( 4 per boar). The boxes enclose the 25 th and 75 th percentiles; the line is the median; and the whiskers extend to the 5th and 95th percentiles. Data are the means \pm SEM of 4 semen samples per boar. 